\title{
Greedy Algorithms for Nonnegativity-Constrained Simultaneous Sparse Recovery
}

\author{
Daeun Kim, Justin P. Haldar* \\ Signal and Image Processing Institute, Ming Hsieh Department of Electrical Engineering, University of Southern California, \\ Los Angeles, CA, 90089 USA
}

\begin{abstract}
This work proposes a family of greedy algorithms to jointly reconstruct a set of vectors that are (i) nonnegative and (ii) simultaneously sparse with a shared support set. The proposed algorithms generalize previous approaches that were designed to impose these constraints individually. Similar to previous greedy algorithms for sparse recovery, the proposed algorithms iteratively identify promising support indices. In contrast to previous approaches, the support index selection procedure has been adapted to prioritize indices that are consistent with both the nonnegativity and shared support constraints. Empirical results demonstrate for the first time that the combined use of simultaneous sparsity and nonnegativity constraints can substantially improve recovery performance relative to existing greedy algorithms that impose less signal structure.
\end{abstract}

Keywords: Compressed Sensing, Simultaneous sparsity, Nonnegativity, Greedy Algorithms

\section{Introduction}

We consider the following inverse problem: given data matrix $\mathbf{Y} \in \mathbb{R}^{M \times K}$ (possibly noisy), observation/dictionary matrix $\boldsymbol{\Phi} \in \mathbb{R}^{M \times N}$, and noiseless linear measurement model $\mathbf{Y}=\mathbf{\Phi X}$, we seek to recover the unknown matrix $\mathbf{X} \in \mathbb{R}^{N \times K}$ by solving the optimization problem:

$$
\hat{\mathbf{X}}=\underset{\mathbf{X} \in \Omega}{\arg \min }\|\mathbf{Y}-\mathbf{\Phi} \mathbf{X}\|_{F}^{2},
$$

\footnotetext{
* Corresponding author

Email address: jhaldar@usc.edu (Justin P. Haldar)
} 
In this work, we propose new greedy algorithms for solving $P_{\Omega}$ under two specific constraints: the columns of $\mathbf{X}$ are both nonnegative (NN) and simultaneously sparse (SS). Specifically, we derive combined NN and SS (NNS) extensions of the following existing greedy algorithms for sparse recovery: orthogonal matching pursuit (OMP) [1 4, subspace pursuit (SP) [5], CoSaMP [6], and hard thresholding pursuit (HTP) [7. The proposed extensions are also easily generalized to other greedy sparse algorithms like iterative hard thresholding (IHT) [8] which are based on similar principles. Applications where these constraints and algorithms may prove useful include magnetic resonance relaxometry [9, 10, kinetic parameter estimation in dynamic positron emission tomography [11, spectral unmixing [12], and sparse NN matrix factorization [13].

Existing work has already demonstrated that combining sparsity constraints with NN constraints leads to improved reconstruction results, both when using convex relaxations [14] and when using greedy algorithms 15. Extensions of greedy sparse recovery algorithms such as OMP, SP, and HTP to the SS context also already exist [16 24]. However, to the best of our knowledge, there are no previously-proposed algorithms for combining NN with SS. This paper fills that gap by proposing a new family of greedy NNS algorithms, and by demonstrating empirically that combined NNS constraints can substantially improve recovery performance relative to the individual use of $\mathrm{NN}$, sparsity, or SS constraints.

This paper is organized as follows. Prior work related to NN constraints and SS constraints is discussed in Section 2. The details of our proposed NNS greedy algorithms are described in Section 3 . Theoretical considerations for the proposed algorithms are discussed in Section 4 . The simulations we use to evaluate the proposed algorithms are described in Section 5 , while the corresponding results are presented and analyzed in Section 6. An application example is described in Section 7. Finally, we provide additional discussion in Section 8 and conclusions in Section 9

\section{Background}

\subsection{Nonnegativity Constraints}

Formally, we define the set of NN signals as

$$
\Omega_{+} \triangleq\left\{\begin{aligned}
\mathbf{X} \in \mathbb{R}^{N \times K}: x_{n k} \geq 0, & n=1, \ldots, N \\
k & =1, \ldots, K
\end{aligned}\right\},
$$

where $x_{n k}$ is the entry in the $n$th row and $k$ th column of $\mathbf{X}$. NN signals are encountered in a variety of applications, due to the fact that certain physical and mathematical quantities are inherently NN. For instance, Euclidean distances, image intensities, signal powers, probabilities, photon counts, and volume fractions are all examples of positive-valued quantities, with negative values being unphysical and difficult to interpret. The use of NN constraints is therefore essential in certain applications, and has a long history: see 25] for review. Interestingly, it has been demonstrated theoretically that NN constraints alone can lead to unique and robust solutions to $P_{\Omega}$ when $\boldsymbol{\Phi}$ is underdetermined, under appropriate additional conditions on $\boldsymbol{\Phi}$ and $\mathbf{Y}$ [26 28]. In addition, the NN least squares (NNLS) problem (the common name for $P_{\Omega}$ combined with constraint set $\Omega_{+}$) is a simple convex optimization problem for which efficient algorithms already exist [25]. 


\subsection{Sparsity and Simultaneous Sparsity Constraints}

Single measurement vector (SMV) sparsity (i.e., sparsity for the case when $K=1$ ) has also emerged as a popular constraint for solving $P_{\Omega}$ in underdetermined settings. This popularity is based on three main observations: (i) most real-world signals possess structure that allows them to be sparsely represented in an appropriate basis or frame; (ii) if $\mathbf{X}$ is sufficiently sparse and the underdetermined $\mathbf{\Phi}$ matrix has appropriate subspace structure, then various sparsity-constrained solutions to $P_{\Omega}$ are theoretically guaranteed to yield stable and accurate estimates of $\mathbf{X}$ [29]; and (iii) even when theoretical guarantees are not applicable, the use of appropriate sparsity constraints allows valuable prior information to be incorporated into the estimation process, generally yielding better results than an unconstrained reconstruction would.

We define the set of $S$-sparse SMV signals as

$$
\Omega_{S} \triangleq\left\{\mathbf{X} \in \mathbb{R}^{N \times 1}:\left|\Lambda_{S}(\mathbf{X})\right| \leq S\right\},
$$

where the support set $\Lambda_{S}(\mathbf{X})$ is defined as

$$
\Lambda_{S}(\mathbf{X}) \triangleq\left\{n \in\{1, \ldots, N\}: x_{n 1} \neq 0\right\}
$$

and $\left|\Lambda_{S}(\mathbf{X})\right|$ denotes its cardinality. The cardinality of this set equals the number of nonzeros in $\mathbf{X}$, such that $\Omega_{S}$ is the set of all vectors that possess no more than $S$ nonzero entries.

Rather than just considering standard SMV sparsity, this work focuses on SS, a specific form of structured MMV sparsity (SS is also sometimes called joint sparsity, group sparsity, or multi-channel sparsity). In SS, it is assumed that the columns of $\mathbf{X}$ are each sparse and share a common support set. Formally, we define the set of simultaneously $S$-sparse signals as

$$
\Omega_{S S} \triangleq\left\{\mathbf{X} \in \mathbb{R}^{N \times K}:\left|\Lambda_{S S}(\mathbf{X})\right| \leq S\right\},
$$

where the shared support set $\Lambda_{S S}(\mathbf{X})$ is defined as

$$
\Lambda_{S S}(\mathbf{X}) \triangleq\left\{n \in\{1, \ldots, N\}: x_{n k} \neq 0 \text { for some } k\right\}
$$

The cardinality of $\Lambda_{S S}(\mathbf{X})$ is equal to the number of rows of $\mathbf{X}$ that are not identically zero, such that $\Omega_{S S}$ is the set of all matrices $\mathbf{X} \in \mathbb{R}^{N \times K}$ possessing no more than $S$ nonzero rows. Unsurprisingly, methods that impose SS are empirically more powerful than methods that solely impose sparsity without enforcing structured sparsity information [30.

Unlike the easy-to-solve NNLS optimization problem 11 sparsity-constrained optimization problems generally have combinatorial complexity. As a result, it is common in the literature to either consider greedy algorithms [16, 20, 22, 24] or convex / nonconvex relaxations of the sparsity constraint [17, 18, 32, 35]. We focus on greedy approaches.

\footnotetext{
${ }^{1}$ Interestingly, the classical active-set NNLS algorithm of Lawson and Hansen 31 has strong algorithmic similarities to NN-OMP [15], a greedy algorithm designed for solving $P_{\Omega}$ with $\Omega=\Omega_{+} \cap \Omega_{S}$. However, while the active-set NNLS algorithm is guaranteed to optimally solve $P_{\Omega}$, the optimality of the result produced by NN-OMP depends on the characteristics of $\boldsymbol{\Phi}$.
} 


\subsection{Greedy Sparse Algorithms}

While greedy optimization algorithms can potentially be trapped at suboptimal local minima, they are frequently less computationally demanding than relaxation-based algorithms, and several have optimality guarantees under appropriate conditions on $\boldsymbol{\Phi}[2,4,8,15,16,18,20,22,24,29,30$. Unlike relaxation-based methods, greedy algorithms attempt to provide solutions that minimize the original (unrelaxed) problem formulation $P_{\Omega}$, and can also benefit from prior knowledge of the sparsity level $S$. Due in part to these features, there are several cases in which greedy algorithms have been observed to empirically outperform relaxation-based approaches [5, 7, 8, 18, 22, 36.

Assuming $S$ is known, greedy algorithms that promote SMV sparsity [1, 2, 4, 8, typically alternate between two steps in an iterative fashion:

1. Based on the estimates of the matrix $\hat{\mathbf{X}}^{(j-1)}$ and support set $\hat{\Lambda}_{S}^{(j-1)}$ from the previous iteration, generate an updated support estimate $\hat{\Lambda}_{S}^{(j)}$.

2. Generate an updated estimate of $\mathbf{X}$ according to

$$
\hat{\mathbf{X}}^{(j)}=\underset{\substack{\mathbf{X} \in \mathbb{R}^{N \times K} \\ \Lambda_{S}(\mathbf{X}) \subseteq \hat{\Lambda}_{S}^{(j)}}}{\arg \min }\|\mathbf{Y} \mathbf{X}\|_{F}^{2} .
$$

Eq. (6) is a simple LLS problem that is generally overdetermined when $M>\left|\hat{\Lambda}_{S}^{(j)}\right|$, and is thus easily and uniquely solved using standard LLS solvers.

The iterative process is terminated once a suitable convergence criteria is met. Most greedy SS algorithms [16 24] follow a similar procedure, replacing $\hat{\Lambda}_{S}^{(j)}$ with $\hat{\Lambda}_{S S}^{(j)}$.

The main differences between different greedy algorithms occur in the support estimation step. We will focus attention on two classes of greedy algorithms: algorithms based on residual correlation and algorithms based on iterative thresholding. In this section, we will concentrate on qualitative descriptions of these algorithms, leaving specific details to the corresponding references. We will also use the notation we have introduced for the SS case, since the SS algorithms typically reduce to their SMV counterparts when $K=1$. Without loss of generality, the rest of the paper will also assume that $\mathbf{\Phi}$ has been normalized to have unitnorm columns, which greatly simplifies notation. Note that the support set of the optimal solution to $P_{\Omega}$ does not change because of column normalization, and that the solutions to $P_{\Omega}$ without column normalization can generally be obtained by simple rescaling of the solutions to $P_{\Omega}$ obtained with normalized columns.

\subsubsection{Residual Correlation Algorithms}

Methods like OMP, SP, CoSaMP, and their variations [1, 2, 4, 6, 15, 20, 22, 24, use residual correlation metrics to update the estimated support set. Specifically, OMP and Simultaneous OMP (S-OMP) [1, 2, 4, 16 20] are stepwise forward selection methods that start with $\hat{\Lambda}_{S S}^{(0)}=\emptyset$, where $\emptyset$ denotes the empty set. Subsequently, the index set is updated as $\hat{\Lambda}_{S S}^{(j)}=\hat{\Lambda}_{S S}^{(j-1)} \cup \lambda^{(j)}$, with

$$
\lambda^{(j)}=\underset{i \in\{1, \ldots, N\}}{\arg \max } \mu\left(\boldsymbol{\Phi}_{i}, \mathbf{R}^{(j-1)}\right),
$$


where

$$
\mu\left(\boldsymbol{\phi}_{i}, \mathbf{R}^{(j-1)}\right) \triangleq\left(\sum_{k=1}^{K}\left|\left\langle\mathbf{r}_{k}^{(j-1)}, \boldsymbol{\phi}_{i}\right\rangle\right|^{p}\right)^{1 / p}
$$

1 is the residual correlation function, $\boldsymbol{\Phi}_{i}$ is the $i$ th column of $\boldsymbol{\Phi}, \mathbf{R}^{(j-1)}=\left(\mathbf{Y}-\mathbf{\Phi} \hat{\mathbf{X}}^{(j-1)}\right)$ is the residual for the previous iteration, $\mathbf{r}_{k}^{(j-1)}$ is the $k$ th column of $\mathbf{R}^{(j-1)}$, and $p$ is typically chosen as 1,2 , or $\infty$. The algorithm terminates after $S$ iterations have been completed, or once $\mathbf{R}^{(j)}$ becomes small. OMP and related methods monotonically decrease the cost function of $P_{\Omega}$, and have small computational cost due to the finite number of iterations. Under conditions on $\boldsymbol{\Phi}$, these algorithms also have theoretical guarantees [1, 2, 4, 16, 20.

These algorithms use (7) to identify the column of $\boldsymbol{\Phi}$ that is most highly-correlated with the current residual, which is subsequently added to the support estimate. When $K=1$ or $p=2$, this choice is optimal in a greedy sense [17], with

$$
\lambda^{(j)}=\underset{i \in\{1, \ldots, N\}}{\arg \min }\left\{\min _{\mathbf{x} \in \mathbb{R}^{1 \times K}}\left\|\boldsymbol{\Phi}_{i} \mathbf{x}-\mathbf{R}^{(j-1)}\right\|_{F}^{2}\right\} .
$$

When $K>1$, other choices of $p$ can also be theoretically justified [16, 18, 20].

Similar to OMP and S-OMP, algorithms like SP, CoSaMP, and Simultaneous CoSaMP (S-CoSaMP) [5, 6, 24] also use residual correlations to determine which support indices to add to $\hat{\Lambda}_{S S}^{(j)}$. However, SP, CoSaMP, and S-CoSaMP are distinct because they propose sets of multiple candidate support indices $\Gamma^{(j)}$ to add to $\hat{\Lambda}_{S S}^{(j)}$ in each iteration, instead of just adding a single support index. Specifically, given an algorithmspecific fixed integer $Q$ (equal to $K$ for SP and S-SP, and $2 K$ for CoSaMP and S-CoSaMP), these algorithms choose $\Gamma^{(j)}$ to include the indices of the $Q$ columns of $\boldsymbol{\Phi}$ that yield the $Q$ largest values of the residual correlation $\mu\left(\boldsymbol{\Phi}_{i}, \mathbf{R}^{(j-1)}\right)$. Since $\hat{\Lambda}_{S S}^{(j-1)} \cup \Gamma^{(j)}$ will have more than $K$ support indices, a trimming procedure is used to remove indices that have the smallest contribution to the solution. The capability to remove support indices from $\hat{\Lambda}_{S S}^{(j)}$ gives these algorithms the power to correct errors in the support set that were made in previous iterations, and leads to improved theoretical performance over algorithms like OMP and S-OMP [5, 6, 24]. However, these algorithms do not necessarily monotonically decrease the cost function of $P_{\Omega}$ or converge for arbitrary $\boldsymbol{\Phi}$. SP has guaranteed finite convergence under specific conditions on $\boldsymbol{\Phi}[5]$.

\subsubsection{Iterative Thresholding Algorithms}

Iterative thresholding algorithms like HTP, IHT, Simultaneous HTP (S-HTP), and Simultaneous IHT (S-IHT) [7, 8, 21, 24, follow a different support-identification strategy. Specifically, given the $\hat{\mathbf{X}}^{(j-1)}$ from the previous iteration, these algorithms use simple gradient descent to identify a matrix $\tilde{\mathbf{G}}^{(j)}$ that is close to $\hat{\mathbf{X}}^{(j-1)}$ but has better data consistency with the measurements $\mathbf{Y}$. In general, this $\tilde{\mathbf{G}}^{(j)}$ is no longer sparse. To identify a sparse support estimate, $\hat{\Lambda}_{S S}^{(j)}$ is chosen to be the set of indices for the $S$ rows of $\tilde{\mathbf{G}}^{(j)}$ with the largest Frobenius norms. This choice is optimal in the sense that

$$
\hat{\Lambda}_{S S}^{(j)}=\underset{\substack{\Lambda \subseteq\{1, \ldots, N\} \\|\Lambda|=S}}{\arg \min }\left\{\min _{\substack{\mathbf{X} \in \mathbb{R}^{N \times K} \\ \Lambda_{S S}(\mathbf{X}) \subseteq \Lambda}}\left\|\mathbf{X}-\tilde{\mathbf{G}}^{(j)}\right\|_{F}^{2}\right\} .
$$




\subsection{NNS Greedy Algorithms Based on Residual Correlation}

The previously described algorithms based on residual correlation used (8) to select candidate support indices. However, it should be noted that (8) ignores the sign of the inner product between the residual and each $\boldsymbol{\phi}_{i}$. The sign of the inner product is highly relevant when considering NN constraints, because $\boldsymbol{\Phi}_{i}$ that are negatively correlated with the residual will generally require negative coefficients in order to reduce the residual. As a result, we use a new definition of residual correlation that is specifically adapted to NN:

$$
\mu_{n n}\left(\boldsymbol{\Phi}_{i}, \mathbf{R}^{(j-1)}\right) \triangleq\left(\sum_{k=1}^{K}\left|\left[\left\langle\mathbf{r}_{k}^{(j-1)}, \boldsymbol{\Phi}_{i}\right\rangle\right]_{+}\right|^{p}\right)^{1 / p},
$$

where $[\cdot]_{+}$is an operator that sets all of the negative entries of its argument to zero, and can be viewed as a sign-based hard thresholding operator. This choice was motivated by similarities to the OMP/S-OMP

\footnotetext{
${ }^{2}$ Note that our formulation assumes that the signal coefficients are NN and SS in the same domain. Our proposed algorithms do not apply to cases where the signal coefficients are NN in one transform domain and SS in another.
} 
support index selection procedure. Specifically, if $K=1$ or $p=2$, then

$$
\lambda^{(j)}=\underset{i \in\{1, \ldots, N\}}{\arg \min }\left\{\min _{\substack{\mathbf{x} \in \mathbb{R}^{1 \times K} \\ \mathbf{x} \geq 0}}\left\|\boldsymbol{\Phi}_{i} \mathbf{x}-\mathbf{R}^{(j-1)}\right\|_{F}^{2}\right\}
$$

1 has the same optimal solution as

$$
\lambda^{(j)}=\underset{i \in\{1, \ldots, N\}}{\arg \max } \mu_{n n}\left(\boldsymbol{\Phi}_{i}, \mathbf{R}^{(j-1)}\right) .
$$




The NNS-CoSaMP algorithm is obtained by making two changes to Alg. 2. First, the size of $\Gamma^{(j)}$ should be changed from $S$ to $2 S$ in step 1 . And second, $\mathbf{X}^{(j)}$ is updated in step 5 by setting all of its elements equal to zero except for those contained in the refined set $\hat{\Lambda}_{S S}^{(j)}$.

Our proposed NNS greedy algorithms were each obtained by making simple modifications to existing sparse greedy algorithms to prioritize and enforce NN structure. For example, the original OMP/S-OMP algorithms [1, 4, 16, 20] are obtained by replacing the references to $\mu_{n n}\left(\boldsymbol{\Phi}_{i}, \mathbf{R}^{(j-1)}\right)$, (14), and (11) in Alg. 1 with references to $\mu\left(\boldsymbol{\Phi}_{i}, \mathbf{R}^{(j-1)}\right),(7)$, and (6), respectively. The original SP algorithm [5] is obtained by making similar substitutions in Alg. 2 when $K=1$. The original S-CoSaMP algorithm [24] is obtained by making these same modifications in Alg. 2, while also replacing $S$ with $2 S$ in step 1 and changing the update procedure in step 5. Clearly, NNS-constrained variations of other greedy algorithms based on residual correlation could be easily obtained by making similar modifications.

It should be noted that the Karush-Kuhn-Tucker necessary conditions for the optimality of $\hat{\mathbf{X}}^{(j-1)}$ in (11) require that $\boldsymbol{\phi}_{\ell}^{T} \mathbf{r}_{k}^{(j-1)} \leq 0$ for each $\ell \in \hat{\Lambda}_{S S}^{(j-1)}$ and each $k$ [25]. As a result, the indices in $\lambda^{(j)}$ or $\Gamma^{(j)}$ in these two algorithms will never duplicate indices that already exist in $\hat{\Lambda}_{S S}^{(j-1)}$.

There are multiple choices for stopping criteria for the NNS-SP algorithm. The original SP algorithm [5] stopped if the norm of the residual increased at any iteration, which ensures monotonicity and finite termination of iterations. This is the stopping criterion that we use in the simulation experiments we describe later in the paper. Other reasonable choices include stopping once a maximum number of iterations has been reached, once the norm of the residual becomes small, or once the iterations stagnate.

\subsection{NNS Greedy Algorithms Based on Iterative Thresholding}

The previously described greedy algorithms based on iterative thresholding used the Frobenius norms of the rows of $\tilde{\mathbf{G}}^{(j)}$ to determine $\hat{\Lambda}_{S S}^{(j)}$. However, this choice ignores the signs of the entries of $\tilde{\mathbf{G}}^{(j)}$, and can be suboptimal in the presence of NN constraints. To rectify this situation, we propose to determine $\hat{\Lambda}_{S S}^{(j)}$ by selecting the rows of $\left[\tilde{\mathbf{G}}^{(j)}\right]_{+}$with the largest Frobenius norms. Incorporating this sign-based hard thresholding operator in the decision process ensures that negative values of $\tilde{\mathbf{G}}^{(j)}$ will not have an undue influence on the selection of $\hat{\Lambda}_{S S}^{(j)}$, and is optimal in the sense that

$$
\hat{\Lambda}_{S S}^{(j)}=\underset{\substack{\Lambda \subseteq\{1, \ldots, N\} \\|\Lambda|=S}}{\arg \min }\left\{\min _{\substack{\mathbf{X} \in \Omega_{+} \\ \Lambda_{S S}(\mathbf{X}) \subseteq \Lambda}}\left\|\mathbf{X}-\tilde{\mathbf{G}}^{(j)}\right\|_{F}^{2}\right\} .
$$

Eq. 15 matches (10), except that it incorporates the NN constraint. Using this support selection rule to modify the normalized HTP algorithm [7], we arrive at the NNS-HTP algorithm described in Alg. 3. 


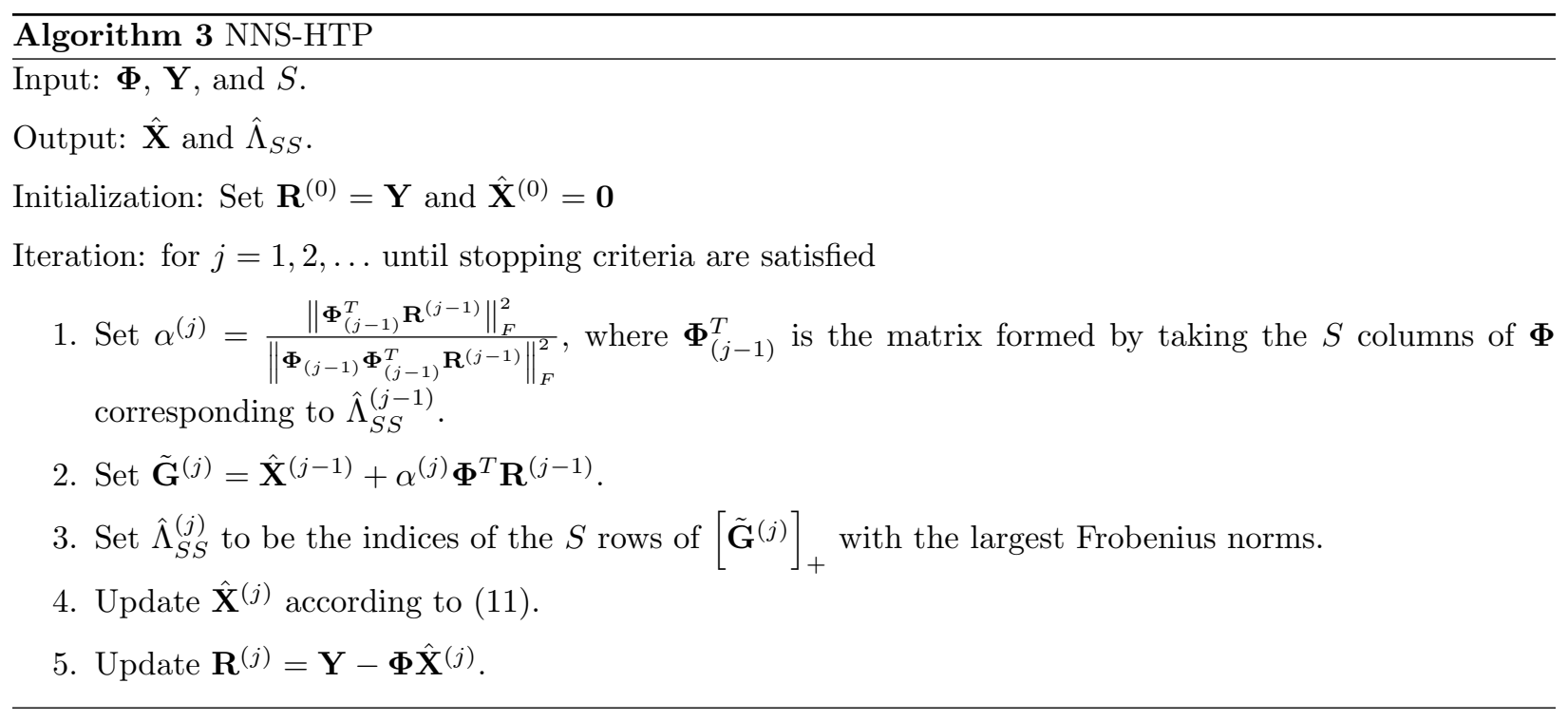

As in the previous cases, our proposed NNS-HTP is a small modification of the existing normalized HTP and simultaneous normalized HTP (S-HTP) algorithms 17, 24], and the original algorithms can be obtained by removing $[\cdot]_{+}$from Alg. 3 and replacing references to (11) with references to (6).

Stopping criteria for NNS-HTP can be chosen in exactly the same way as the stopping criteria for NNS-SP. In the simulation experiments described below, we stop the iterations if the norm of the residual increases, which imposes monotonicity on the proposed algorithms.

\section{Theoretical Considerations}

The previous sections described our proposed NNS greedy algorithms. However, we have not focused in this paper on important theoretical questions like those given below.

1. Under what conditions does $P_{\Omega}$ with $\Omega=\Omega_{+} \bigcap \Omega_{S S}$ have a unique solution?

2. Under what conditions does the solution to $P_{\Omega}$ produce a good approximation of the original $\mathbf{X}$ ?

3. Under what conditions will an NNS greedy algorithm obtain an optimal solution to $P_{\Omega}$ ?

We would like to point out that good answers to these questions can be obtained by combining theoretical results from the existing literature. For example, there is a substantial body of literature that has already established uniqueness and correctness guarantees for the solution to $P_{\Omega}$ when the constraints $\Omega_{+}$and $\Omega_{S S}$ are used individually [15] 24, $[26,28,3$ As a result, conditions for the uniqueness and correctness of the solution to $P_{\Omega}$ with $\Omega=\Omega_{+} \bigcap \Omega_{S S}$ can be trivially obtained by taking a simple union of the conditions for uniqueness and correctness of the solution to $P_{\Omega}$ under $\Omega_{+}$and $\Omega_{S S}$ individually. While this does not

\footnotetext{
${ }^{3}$ Due to space constraints and the diversity of different conditions that lead to guaranteed uniqueness and correctness, we make no attempt to summarize these different conditions. Interested readers are encouraged to examine Refs. [15-24] 26- 28].
} 

and accurately solved in a large range of different scenarios.

It is also straightforward to guarantee the performance of various NNS greedy algorithms by making simple modifications to the performance guarantee proofs for existing greedy algorithms. For example, using techniques similar to those described in 2, 19, we have derived a performance guarantee for NNS-OMP based on mutual coherence [2], which is one of the mathematical characteristics of $\boldsymbol{\Phi}$ that is frequently used to guarantee the success of sparse recovery methods. Specifically, based on the mutual coherence definition

$$
\mu\{\boldsymbol{\Phi}\} \triangleq \max _{\substack{i, j \in\{1, \ldots, N\} \\ i \neq j}}\left|\left\langle\boldsymbol{\Phi}_{i}, \boldsymbol{\Phi}_{j}\right\rangle\right|
$$

we can prove the following theorem:

Theorem 1. Let $\mathbf{Y}=\mathbf{\Phi} \mathbf{X}^{\mathrm{opt}}$ for fixed $\mathbf{Y} \in \mathbb{R}^{M \times K}, \mathbf{X}^{\mathrm{opt}} \in \mathbb{R}^{N \times K}$, and $\mathbf{\Phi} \in \mathbb{R}^{M \times N}$ with unit-norm columns. If $\mathbf{X}^{\mathrm{opt}} \in \Omega_{+}, S=\left|\Lambda_{S S}\left(\mathbf{X}^{\mathrm{opt}}\right)\right|$, and

$$
S<\min \left\{\frac{1}{2}\left(1+\frac{1}{\mu\{\boldsymbol{\Phi}\}}\right), 1+\frac{1}{0.5 K+\mu\{\boldsymbol{\Phi}\}}\right\},
$$

then NNS-OMP with $p=1$ is guaranteed to reconstruct $\mathbf{X}^{\mathrm{opt}}$ exactly in $S$ steps.

The proof of this theorem is given in the supplementary material accompanying this paper. It should be noted that the condition on mutual coherence in $(17)$ is rather restrictive, and that the original OMP would be theoretically guaranteed to perfectly recover an NNS signal under less restrictive conditions. However, as our simulation results illustrate, NNS-OMP works very well in many cases where the mutual coherence condition is violated. We anticipate that less-restrictive performance guarantees for this and other NNS greedy algorithms are possible to construct, but leave such effort to future work.

\subsection{Practical Coherence Considerations}

For practical problems involving NN constraints, the matrix $\mathbf{\Phi}$ frequently does not have good mutual coherence properties [15] (e.g., $\mu \approx 0.9999$ for our HARDI example in Section 7). In [15, Bruckstein et al. applied a "conditioning" procedure that reduces the mutual coherence of the matrix $\mathbf{\Phi}$. As expected, reducing the mutual coherence frequently improved the empirical performance of NN-OMP in [15]. Our preliminary experience suggests that such conditioning also frequently improves the empirical performance of the NNS greedy algorithms proposed in this work. For cases when $\boldsymbol{\Phi}$ is a non-negative matrix, Bruckstein et al. suggested replacing $\mathbf{Y}=\mathbf{\Phi} \mathbf{X}$ with the linear model $\mathbf{C Y}=\mathbf{C \Phi X}$, where $\mathbf{C} \in \mathbb{R}^{M \times M}$ is the nonsingular matrix defined by $\mathbf{C} \triangleq \mathbf{I}-\frac{1-\epsilon}{M} \mathbf{1}$, where $\mathbf{I}$ is the $M \times M$ identity matrix, $\mathbf{1}$ is the $M \times M$ matrix of all ones, and $\epsilon$ is a small positive constant with $0<\epsilon \ll 1$. The effects of this conditioning procedure on our proposed algorithms will be explored empirically in Section 6.2 


\section{Simulation Setup}

Several numerical experiments were designed to evaluate the performance of the NNS-greedy algorithms relative to algorithm variations that impose less signal structure. Specifically, we compare the NNS greedy algorithms (i.e., NNS-OMP, NNS-SP, NNS-CoSaMP, and NNS-HTP) against: the original greedy algorithms with neither NN nor SS constraints (i.e., OMP, SP, CoSaMP, and HTP, with the algorithms applied to each column of $\mathbf{Y}$ independently), the NN-only versions of the algorithms (e.g., NN-OMP, NN-SP, NN-CoSaMP, and NN-HTP, with the algorithms applied to each column of $\mathbf{Y}$ independently), and the SS-only versions of the algorithms (e.g., S-OMP, S-SP, S-CoSaMP, and S-HTP). All algorithms for this comparison were implemented in MATLAB (Mathworks Inc., Natick, MA), and we used Lawson and Hanson's active-set NNLS algorithm [31] (MATLAB's Isqnonneg function) to solve 111.

In each experiment, the values of $M, N, K$, and $S$ were predetermined, and corresponding matrices $\mathbf{\Phi} \in \mathbb{R}^{M \times N}, \mathbf{X} \in \mathbb{R}^{N \times K}$, and $\mathbf{Y} \in \mathbb{R}^{M \times K}$ were generated randomly. Specifically:

- In one set of experiments, the entries of each $\boldsymbol{\Phi}$ matrix were sampled randomly from a standard independent and identically distributed (iid) Gaussian distribution. In a second set of experiments, the entries of each $\boldsymbol{\Phi}$ matrix were sampled randomly from an iid Rayleigh distribution, yielding a nonnegative matrix. Nonnegative dictionaries are common in practical applications, and Rayleigh matrices would be expected to have worse mutual coherence values than Gaussian matrices.

- The support set $\Lambda_{S S}(\mathbf{X})$ of each $\mathbf{X}$ matrix was chosen as the first $S$ values from a random permutation of the integers between 1 and $N$. Rows belonging to the support set were then populated by sampling iid from one of two different probability distributions: the Rayleigh distribution and the Uniform $[0,1]$ distribution. Both of these distributions yield samples that are NN, ensuring that $\mathbf{X} \in \Omega_{+} \bigcap \Omega_{S S}$.

- Noiseless measured data $\mathbf{Y}$ was generated according to $\mathbf{Y}=\boldsymbol{\Phi} \mathbf{X}$. We also generated noisy measured data by adding an iid Gaussian noise matrix $\mathbf{N} \in \mathbb{R}^{M \times K}$ to $\mathbf{Y}$. For this work, we define the signal-tonoise ratio (SNR) of noisy $\mathbf{Y}$ matrices as $\|\mathbf{\Phi} \mathbf{X}\|_{F}$ divided by the expected value of $\|\mathbf{N}\|_{F}$.

\section{Simulation Results}

\subsection{Gaussian $\mathbf{\Phi}$ Matrices}

The simulations described in this subsection all use iid Gaussian $\mathbf{\Phi}$ matrices, which are expected to have relatively good mutual coherence properties. Our first set of simulations explored the performance of the different greedy algorithms as a function of $K$, in a noiseless setting with $M=24, N=128, p=2$, and fixed sparsity level $S=4$. The simultaneous level $K$ was systematically varied between 1 and 30 . For each value of $K$, the simulation was repeated 5000 times with different realizations of $\mathbf{X}$ and $\mathbf{\Phi}$. Figure 1 shows the results of these simulations, plotting the perfect recovery probability as a function of $K$. We say that perfect recovery has occurred for a given problem realization whenever the infinity norm of the reconstruction error 
$\|\mathbf{X}-\widehat{\mathbf{X}}\|_{\infty}$ is less than $10^{-4}$. We estimate the probability of perfect recovery by taking ratio between the number of perfect recoveries and the total number of trials. The plots also include a $95 \%$ confidence interval for the probability of perfect recovery, which we computed using the Wald test [37. The results indicate that algorithms that use NNS or SS constraints have better performance than algorithms that do not use any form of SS constraints, and that the performance gap increases as the simultaneous level $K$ increases 4 This is not surprising, since the SS constraint provides valuable prior information that is not fully captured by NN or unstructured sparsity constraints. As expected, we also observe that the NNS algorithms outperform the algorithms based on SS constraints alone.

While the perfect recovery probabilities were not identical for $\mathbf{X}$ matrices generated using the Rayleigh and Uniform probability densities, the general performance trends we observed were similar. This was also true for our other simulations, and for simplicity, we only show Rayleigh $\mathbf{X}$ in what follows.

Our second set of simulations explored the performance of our proposed algorithms in the presence of noise. Simulation parameters were identical to the first simulation, except that we fixed $K=8$ and added noise with SNR values ranging from $0 d B$ to $100 d B$. Since perfect recovery is generally not possible in the noisy case, we measured performance by estimating the probability of perfect support recovery. We say that perfect support recovery has occurred for a given problem realization if $\hat{\Lambda}_{S S}=\Lambda_{S S}(\mathbf{X})$. Plots of the perfect support recovery probability versus SNR are shown in Fig. 2. We observe that algorithm performance decreases rapidly at lower SNR values $(<60 \mathrm{~dB})$ unless the algorithms use SS constraints. On the other hand, the NNS and SS algorithms still show good performance, with perfect support recovery probabilities higher than 0.75 for SS and higher than 0.9 for NNS when SNR $=10 \mathrm{~dB}$. As in the noiseless case, the proposed NNS algorithms outperform the SS algorithms, sometimes substantially. These simulations demonstrate that the proposed algorithms can still perform well in the presence of substantial noise.

To further explore performance characteristics in the noisy setting, we performed a third set of simulations with SNR fixed at the relatively low value of $10 d B$ and allowing $K$ to vary. All other parameters were identical to those of the previous simulations. Results are shown in Fig. 3, and again demonstrate the value of using both NN and SS constraints together. Our proposed NNS algorithms have better performance than the alternatives. The algorithms based on SS constraints perform comparably well for larger $K$ for certain algorithms (e.g., Fig. 3(b)), though a substantial gap is still present for others (e.g., Fig. 3(d)).

Our previous simulations explored algorithm performance for the relatively small sparsity level of $S=4$. Our fourth and fifth simulations explore algorithm performance as a function of $S$. Both of these simulations used $M=64, N=128, p=2$, and $K=8$, with $S$ systematically varied between 2 and 60 . Each simulation was repeated 1000 times with different realizations of $\mathbf{X}, \mathbf{\Phi}$, and $\mathbf{N}$. The fourth simulation used noiseless data, with results shown in Fig. 4 , while the fifth simulation used noisy data (SNR=30 $d B$ and $10 d B)$, with

\footnotetext{
${ }^{4}$ It should be noted that performance generally gets worse with increasing $K$ for algorithms that do not impose SS sparsity constraints. This behavior is expected, because larger $K$ implies that there are more variables to estimate and more opportunities to estimate one of those variables incorrectly.
} 


\footnotetext{
${ }^{5}$ It should be observed that the mutual coherence values are still relatively large, even in the Gaussian case. For example, with $\mu\{\boldsymbol{\Phi}\}=0.708$, the conditions of Theorem 1 can only be satisfied in the trivial case where $S \leq 1$.
} 
average mutual coherence of $\approx 0.811$.

Figure 7 shows the noiseless recovery performance as a function of $K$. As expected, performance is substantially worse in this case relative to the iid Gaussian case when the conditioning procedure is not applied. In addition, the performance of the NNS greedy algorithms is not very different from the performance of SS greedy algorithms in the absence of conditioning, though the NNS and SS greedy algorithms still substantial outperform the greedy algorithms that only impose NN or unstructured sparsity. Our analysis suggests that the minimal difference between SS and NNS is related to the fact that that the $\mathbf{\Phi}$ and $\mathbf{Y}$ are both NN in this case, leading to substantially more strong positive residual correlations relative to strong negative residual correlations. However, substantial performance improvements for all algorithms are obtained by applying the conditioning procedure, and as before, we observe substantial performance advantages for the NNS greedy algorithms relative to the alternative algorithms that impose less structure.

\section{Application Example}

The previous sections evaluated our proposed methods in the context of simulated data. In this section, we demonstrate the potential benefits of our proposed algorithms in the context of a real-world magnetic resonance imaging (MRI) application. Specifically, we used greedy algorithms to estimate the orientation of fiber bundles from high angular resolution diffusion MRI (HARDI) data 38. HARDI measurements provide information about the random (Brownian) thermal motion of water molecules in the body. This information can be used to infer fiber orientations, since water diffusion is generally anisotropic in fibrous structures, with a tendency for water to displace by longer distances along the principal axis of the fiber [39]. When applied to the human brain, the fiber orientation information can subsequently be used to identify the trajectories of neuronal fiber bundles (the "wires" that carry communications between different brain regions), which provides a form of "wiring diagram" that describes the physical backbone of the brain's internal network.

Within the HARDI literature, an important class of orientation estimation methods models the observed signal as a sparse and nonnegative mixture of basis functions [40 42, where each basis function is a rotated and resampled version of the same basic function, and the prototype function corresponds to the ideal data that would be measured from an ideal fiber bundle. Once the measured data has been decomposed in this basis, the orientation distribution functions (ODFs) that describe the ensemble probability of water displacement along a given orientation can be obtained by applying appropriate transformations to each basis function. An example of several diffusion basis functions and corresponding ODFs is shown in Fig. 8 .

This section applies greedy algorithms to HARDI data with NNS constraints to obtain ODF estimates that reflect the following characteristics:

- The number of distinct fiber bundles that are present in small local region of brain tissue is typically assumed to be small (e.g., 1-3 fiber bundles per voxel [43]). A major benefit of greedy methods is that the sparsity level $S$ can be specified manually, which allows this additional prior information to be injected into the estimation process. 
- Fiber bundles are continuous and generally follow smooth paths through space, which means that fiber orientations are frequently consistent within small local spatial neighborhoods [44. This implies that a SS model may be reasonable for certain brain regions.

- "Negative" fiber bundles are unphysical [40, 42].

Our example uses Fiber Cup diffusion phantom data [45, 46] that was acquired using a diffusion encoding b-value of $2000 \mathrm{~s} / \mathrm{mm}^{2}, M=64$ different measurement orientations, and $3 \mathrm{~mm}$ isotropic spatial resolution. The diffusion phantom consists of water-filled acrylic fiber bundles. We specifically examined a group of $K=128$ voxels from a spatial region where two straight fiber bundles cross at an approximately $90^{\circ}$ angle.

Orientation estimation from HARDI data was mapped into problem $P_{\Omega}$ as described below.

- The data matrix $\mathbf{Y} \in \mathbb{R}^{M \times K}$ was constructed by reformatting the diffusion data from each of $M=64$ measurement orientations and $K=128$ spatial locations into an $M \times K$ matrix.

- The dictionary matrix $\boldsymbol{\Phi} \in \mathbb{R}^{M \times N}$ was obtained by rotating a prototype basis function along each of $N=1296$ orientations. The orientations were designed to be uniformly spaced on the sphere using an electrostatic repulsion model 47. The prototype function was obtained by fitting a spherical harmonic model [48] to data from a spatial region containing a single fiber bundle.

- The unknown (to be estimated) matrix $\mathbf{X} \in \mathbb{R}^{N \times K}$ contains the basis function coefficients, which we know in advance will be sparse and NN.

The matrix $\hat{\mathbf{X}}$ was estimated using the SP algorithms with sparsity level $S=2$ from the conditioned linear model $\mathbf{C Y}=\mathbf{C} \boldsymbol{\Phi X}$, and the results are shown in Fig. 9. As shown in the figure, the proposed NNS-SP algorithm clearly depicts the known geometry of the phantom (two straight fiber bundles that cross at an approximately $90^{\circ}$ angle). In contrast, the NN-SP algorithm yields a field of estimated ODFs that have substantial spatial variability and do not reproduce the known geometry as accurately. In addition, the standard SP and S-SP algorithms produce unphysical negative coefficients for certain voxels, which induces the appearance of unusually large ODFs. Results using OMP, CoSaMP, and HTP-based algorithms were similar, and are not shown. These results demonstrate the potential advantages of our proposed greedy NNS algorithms in real-world applications.

\section{Discussion}

It is worth emphasizing that, while Theorem 1 indicates that performance guarantees can exist for our proposed NNS algorithms, the theory that we have derived is almost completely inapplicable in any of the empirical results we have considered because of the large mutual coherences of the $\boldsymbol{\Phi}$ matrices. Given the structure of most NNS inverse problems, we believe that this is likely to be a widespread occurrence in practical applications. However, we do not view the lack of strong theoretical guarantees as a barrier to the 
use of intelligently designed algorithms that demonstrate good empirical performance. For example, MRI reconstruction from undersampled data is often described as one of the prototypical "success stories" of sparse recovery theory and methods, despite the fact that the existing theoretical performance guarantees are largely inapplicable in these scenarios 49, which means that most good sparsity-based MRI reconstruction results should be considered heuristic rather than theoretically justified. However, whenever theoretical guarantees are absent in the context of a specific application, it is important to perform thorough application-specific testing of the algorithms to help ensure that the algorithms will generate desirable results.

Similar to existing greedy algorithms, many of our proposed algorithms depend on prior knowledge of $S$, which may not always be known in practice. Our preliminary experience indicates that our proposed NNS algorithms can still be very successful and still consistently outperform the less-constrained greedy algorithms in cases when $S$ is overestimated, though a leave more detailed explorations to future work.

It is also worth mentioning that the use of a least-squares data fidelity criterion in $P_{\Omega}$ can be viewed as implicitly using a Gaussian noise model. However, for many applications where NN constraints are reasonable, the data might be more accurately described by the Rician distribution (i.e., each measured data sample is equal to the magnitude of a Gaussian random variable) or the non-central chi distribution (i.e., each measured data sample is equal to the square root of sum-of-squared magnitudes of multiple Gaussian random variables). It should be noted that $P_{\Omega}$ is still relevant to such cases. Specifically, we have recently introduced a quadratic majorize-minimize procedure [50] that allows Rician and noncentral chi log-likelihoods to be maximized by iteratively solving a sequence of NNLS problems, where each NNLS problem has the same form as $P_{\Omega}$. This type of approach is easily adapted to our proposed NNS greedy algorithms.

\section{Conclusions}

This paper proposed a new family of NNS greedy algorithms, and showed empirically that such algorithms can be an effective way to recover NNS signals in ill-conditioned or underdetermined scenarios where the incorporation of accurate additional prior information will generally be expected to enhance reconstruction performance. While we have concentrated primarily on the empirical performance characteristics of these algorithms, we have also proven a performance guarantee for NNS-OMP under mutual coherence conditions on $\mathbf{\Phi}$, and conjecture that better theoretical performance guarantees for these algorithms can also be established. We expect that these algorithms and their extensions will prove useful in a wide variety of applications where NN and SS structure is reasonable to assume.

\section{Acknowledgments}

This work was supported in part by NSF CAREER award CCF-1350563 and NIH grants R01-NS074980 and R01-NS089212. Computation for some of the work described in this paper was supported by the University of Southern California's Center for High-Performance Computing (http://hpcc.usc.edu). 


\section{References}

[1] G. Davis, S. Mallat, M. Avellaneda, Adaptive greedy approximations, Constr. Approx. 13 (1997) 57-98. doi:10.1007/BF02678430.

[2] D. L. Donoho, M. Elad, V. N. Temlyakov, Stable recovery of sparse overcomplete representations in the presence of noise, IEEE Trans. Inf. Theory 52 (2006) 6-18. doi:10.1109/TIT.2005.860430.

[3] J. A. Tropp, A. C. Gilbert, Signal recovery from random measurements via orthogonal matching pursuit, IEEE Trans. Inf. Theory 53 (2007) 4655-4666. doi:10.1109/TIT.2007.909108.

[4] T. T. Cai, L. Wang, Orthogonal matching pursuit for sparse signal recovery with noise, IEEE Trans. Inf. Theory 57 (2011) 4680-4688. doi:10.1109/TIT.2011.2146090.

[5] W. Dai, O. Milenkovic, Subspace pursuit for compressive sensing signal reconstruction, IEEE Trans. Inf. Theory 55 (2009) 2230-2249. doi:10.1109/TIT.2009.2016006.

[6] D. Needell, J. A. Tropp, CoSaMP: Iterative signal recovery from incomplete and inaccurate samples, Appl. Comput. Harmon. Anal. 26 (2009) 301-321. doi:10.1145/1859204.1859229.

[7] S. Foucart, Hard thresholding pursuit: An algorithm for compressive sensing, SIAM J. Numer. Anal. 49 (2011) 2543-2563. doi:10.1137/100806278.

[8] T. Blumensath, M. E. Davies, Normalized iterative hard thresholding: Guaranteed stability and performance, IEEE J. Sel. Topics Signal Process. 4 (2010) 298-309. doi:10.1109/JSTSP.2010.2042411.

[9] K. P. Whittall, A. L. MacKay, Quantitative interpretation of NMR relaxation data, J. Magn. Reson. 84 (1989) 134-152. doi:10.1016/0022-2364(89)90011-5

[10] D. Kumar, T. D. Nguyen, S. A. Gauthier, A. Raj, Bayesian algorithm using spatial priors for multiexponential $T_{2}$ relaxometry from multiecho spin echo MRI, Magn. Reson. Med. 68 (2012) 1536-1543. doi: $10.1002 / \mathrm{mrm} .24170$.

[11] Y. Lin, J. P. Haldar, Q. Li, P. S. Conti, R. M. Leahy, Sparsity constrained mixture modeling for the estimation of kinetic parameters in dynamic PET, IEEE Trans. Med. Imag. 33 (2014) 173-185. doi:10.1109/TMI.2013.2283229.

[12] W.-K. Ma, J. M. Bioucas-Dias, T.-H. Chan, N. Gillis, P. Gader, A. J. Plaza, A. Ambikapathi, C.-Y. Chi, A signal processing perspective on hyperspectral unmixing: Bridging the gap between remote sensing and signal processing, IEEE Signal Process. Mag. 31 (2014) 67-81. doi:10.1109/MSP.2013.2279731.

[13] P. O. Hoyer, Non-negative matrix factorization with sparseness constraints, J. Mach. Learn. Res. 5 (2004) 1457-1469. 
[14] D. L. Donoho, J. Tanner, Sparse nonnegative solution of underdetermined linear equations by linear programming, Proc. Natl. Acad. Sci. USA 102 (2005) 9446-9451. doi:10.1073/pnas.0502269102

[15] A. M. Bruckstein, M. Elad, M. Zibulevsky, On the uniqueness of nonnegative sparse solutions to underdetermined systems of equations, IEEE Trans. Inf. Theory 54 (2008) 4813-4820. doi:10.1109/TIT. 2008.929920 .

[16] D. Leviatan, V. N. Temlyakov, Simultaneous approximation by greedy algorithms, Adv. Comput. Math. 25 (2006) 73-90. doi:10.1007/s10444-004-7613-4.

[17] S. F. Cotter, B. D. Rao, K. Engan, K. Kreutz-Delgado, Sparse solutions to linear inverse problems with multiple measurement vectors, IEEE Trans. Signal Process. 53 (2005) 2477-2488. doi:10.1109/TSP. 2005.849172 .

[18] J. Chen, X. Huo, Theoretical results on sparse representations of multiple-measurement vectors, IEEE Trans. Signal Process. 54 (2006) 4634-4643. doi:10.1109/TSP.2006.881263.

[19] J. A. Tropp, A. C. Gilbert, M. J. Strauss, Algorithms for simultaneous sparse approximation. Part I: Greedy pursuit, Signal Process. 86 (2006) 572-588. doi:10.1016/j.sigpro.2005.05.030

[20] R. Gribonval, H. Rauhut, K. Schnass, P. Vandergheynst, Atoms of all channels, unite! Average case analysis of multi-channel sparse recovery using greedy algorithms, J. Fourier Anal. Appl. 14 (2008) 655-687. doi:10.1007/s00041-008-9044-y.

[21] S. Foucart, Recovering jointly sparse vectors via hard thresholding pursuit, in: Proc. SAMPTA, 2011.

[22] K. Lee, Y. Bresler, M. Junge, Subspace methods for joint sparse recovery, IEEE Trans. Inf. Theory 58 (2012) 3613-3641. doi:10.1109/TIT.2012.2189196.

[23] M. E. Davies, Y. C. Eldar, Rank awareness in joint sparse recovery, IEEE Trans. Inf. Theory 58 (2012) 1135-1146. doi:10.1109/TIT.2011.2173722.

[24] J. D. Blanchard, M. Cermak, D. Hanle, Y. Jing, Greedy algorithms for joint sparse recovery, IEEE Trans. Signal Process. 62 (2014) 1694-1704. doi:10.1109/TSP.2014.2301980.

[25] D. Chen, R. J. Plemmons, Nonnegativity constraints in numerical analysis, in: A. Bultheel, R. Cools (Eds.), The Birth of Numerical Analysis, World Scientific Publishing, 2010, pp. 109-139.

[26] M. Wang, W. Xu, A. Tang, A unique "nonnegative" solution to an underdetermined system: From vectors to matrices, IEEE Trans. Signal Process. 59 (2011) 1007-1016. doi:10.1109/TSP. 2010.2089624.

[27] N. Meinshausen, Sign-constrained least squares estimation for high-dimensional regression, Electron. J. Statist. 7 (2013) 1607-1631. doi:10.1214/13-EJS818. 
1 [28] M. Slawski, M. Hein, Non-negative least squares for high-dimensional linear models: Consistency and sparse recovery without regularization, Electron. J. Statist. 7 (2013) 3004-3056. doi:10.1214/ 13-EJS868.

[29] Y. C. Eldar, G. Kutyniok (Eds.), Compressed Sensing: Theory and Applications, Cambridge University Press, 2012.

[30] M. F. Duarte, Y. C. Eldar, Structured compressed sensing: From theory to applications, IEEE Trans. Signal Process. 59 (2011) 4053-4085. doi:10.1109/TSP.2011.2161982.

[31] C. L. Lawson, R. J. Hanson, Solving least squares problems, Vol. 15, SIAM, Philadelphia, 1995.

[32] J. A. Tropp, Algorithms for simultaneous sparse approximation. Part II: Convex relaxation, Signal Process. 86 (2006) 589-602. doi:10.1016/j.sigpro.2005.05.031.

[33] M. Yuan, Y. Lin, Model selection and estimation in regression with grouped variables, J. R. Stat. Soc. Ser. B 68 (2006) 49-67. doi:10.1111/j.1467-9868.2005.00532.x.

[34] M. Fornasier, H. Rauhut, Recovery algorithms for vector-valued data with joint sparsity constraints, SIAM J. Numer. Anal. 46 (2008) 577-613. doi:10.1137/0606668909.

[35] R. Chartrand, B. Wohlberg, A nonconvex ADMM algorithm for group sparsity with sparse groups, in: Proc. IEEE Int. Conf. Acoust., Speech, Signal Process., 2013, pp. 6009-6013. doi:10.1109/ICASSP. 2013.6638818 .

[36] J. P. Haldar, D. Hernando, Rank-constrained solutions to linear matrix equations using PowerFactorization, IEEE Signal Process. Lett. 16 (2009) 584-587. doi:10.1109/LSP.2009.2018223.

[37] F. E. Harrell, Regression modeling strategies: with applications to linear models, logistic regression, and survival analysis, Springer, New York, 2001.

[38] D. S. Tuch, T. G. Reese, M. R. Wiegell, N. Makris, J. W. Belliveau, V. J. Wedeen, High angular resolution diffusion imaging reveals intravoxel white matter fiber heterogeneity, Magn. Reson. Med. 48 (2002) 577-582. doi:10.1002/mrm.10268.

[39] D. LeBihan, H. Johansen-Berg, Diffusion MRI at 25 exploring brain tissue structure and function, NeuroImage 61 (2012) 324-341. doi:10.1016/j.neuroimage.2011.11.006.

[40] A. Ramirez-Manzanares, M. Rivera, B. C. Vemuri, P. Carney, T. Mareci, Diffusion basis functions decomposition for estimating white matter intravoxel fiber geometry, IEEE Trans. Med. Imag. 26 (2007) 1091-1102. doi:10.1109/TMI.2007.900461.

[41] Y. Wang, Q. Wang, J. P. Haldar, F.-C. Yeh, M. Xie, P. Sun, T.-W. Tu, K. Trinkaus, R. S. Klein, A. H. Cross, S.-K. Song, Quantification of increased cellularity during inflammatory demyelination, Brain 134 (2011) 3587 - 3598. doi:10.1093/brain/awr307. 
1 [42] J. Cheng, R. Deriche, T. Jiang, D. Shen, P.-T. Yap, Non-negative spherical deconvolution (NNSD) for estimation of fiber orientation distribution function in single-/multi-shell disffusion MRI, NeuroImage 101 (2014) 750-764. doi:10.1016/j.neuroimage.2014.07.062.

[43] B. Jeurissen, A. Leemans, J.-D. Tournier, D. K. Jones, J. Sijbers, Investigating the prevalence of complex fiber configurations in white matter tissue with diffusion magnetic resonance imaging, Hum. Brain Mapp.

$6 \quad 34$ (2013) 2747 - 2766. doi:10.1002/hbm.22099.

7 [44] A. Raj, C. Hess, P. Mukherjee, Spatial HARDI: Improved visualization of complex white matter architecture with Bayesian spatial regularization, NeuroImage 54 (2011) 396 - 409. doi:10.1016/j. neuroimage.2010.07.040.

[45] P. Fillard, M. Descoteaux, A. Goh, S. Gouttard, B. Jeurissen, J. Malcolm, A. Ramirez-Manzanares, M. Reisert, K. Sakaie, F. Tensaouti, T. Yo, J.-F. Mangin, C. Poupon, Quantitative evaluation of 10 tractography algorithms on a realistic diffusion MR phantom, NeuroImage 56 (2011) 220-234. doi: $10.1016 / j$.neuroimage.2011.01.032.

[46] C. Poupon, B. Rieul, I. Kezele, M. Perrin, F. Poupon, J.-F. Mangin, New diffusion phantom dedicated to the study and validation of high-angular-resolution diffusion imaging (HARDI) models, Magn. Reson. Med. 60 (2008) 1276 - 1283. doi:10.1002/mrm.21789

[47] D. K. Jones, M. A. Horsfield, A. Simmons, Optimal strategies for measuring diffusion in anisotropic systems by magnetic resonance imaging, Magn. Reson. Med. 42 (1999) 515 - 525. doi:10.1002/(SICI) 1522-2594(199909) 42:3<515: :AID-MRM14>3.0.C0;2-Q

[48] M. Descoteaux, E. Angelino, S. Fitzgibbons, R. Deriche, Regularized, fast, and robust analytical Q-ball imaging, Magn. Reson. Med. 58 (2007) 497-510. doi:10.1002/mrm.21277.

[49] J. P. Haldar, D. Hernando, Z.-P. Liang, Compressed-sensing MRI with random encoding, IEEE Trans. Med. Imag. 31 (2011) 893-903. doi:10.1109/TMI.2010.2085084.

[50] D. Varadarajan, J. P. Haldar, A majorize-minimize framework for Rician and non-central chi MR images, IEEE Trans. Med. Imag. 24 (2015) 2191-2202. doi:10.1109/TMI.2015.2427157.

[51] J. P. Haldar, R. M. Leahy, Linear transformation for Fourier data on the sphere: Application to high angular resolution diffusion MRI of the brain, NeuroImage 72 (2013) 233 - 247. doi:10.1016/j. neuroimage.2013.01.022. 


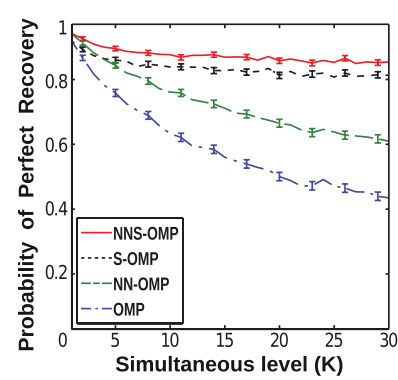

(a) OMP, Rayleigh X



(e) OMP, Uniform X

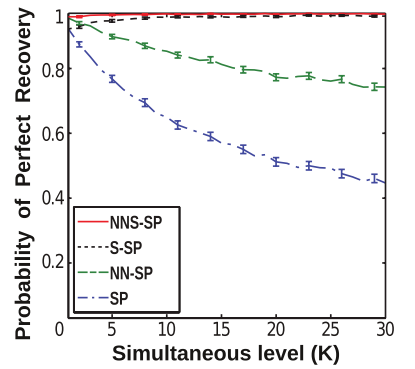

(b) SP, Rayleigh X

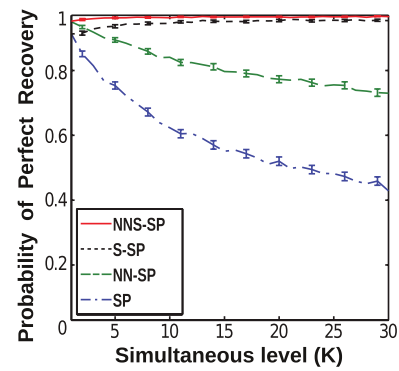

(f) SP, Uniform X



(c) CoSaMP, Rayleigh X

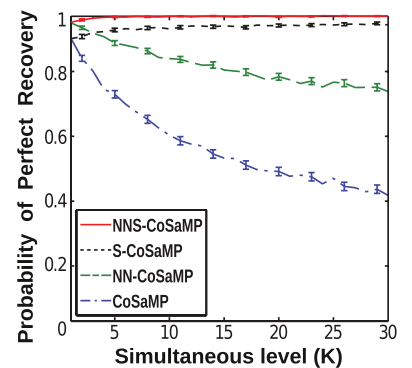

(g) CoSaMP, Uniform X



(d) HTP, Rayleigh X

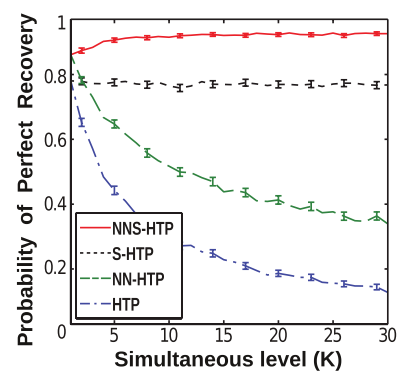

(h) HTP, Uniform X

Figure 1: The empirical probability of perfect recovery as a function of $K$ for the noiseless first set of simulations. Results are plotted for SS X matrices drawn iid from (a)-(d) the Rayleigh distribution and (e)-(f) the Uniform [0, 1] distribution. Results are shown for (a,e) OMP-based algorithms, (b,f) SP-based algorithms, (c,g) CoSaMP-based algorithms, and (d,h) HTP-based algorithms. The error bars in each plot represent $95 \%$ confidence intervals. 


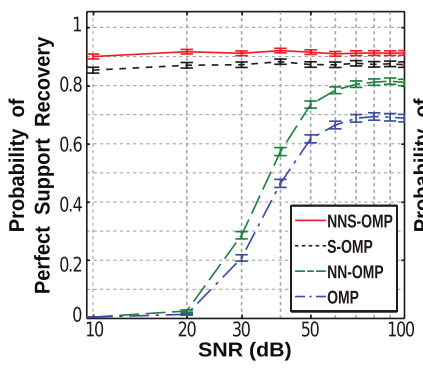

(a) OMP Algorithms

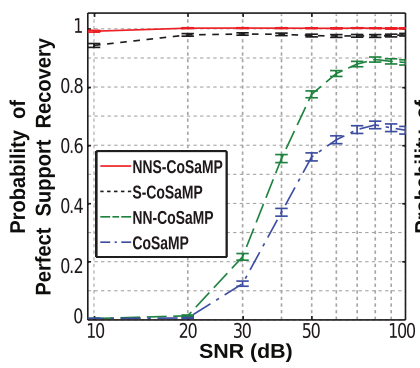

(c) CoSaMP Algorithms

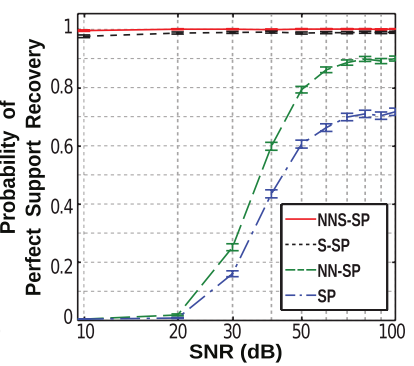

(b) SP Algorithms

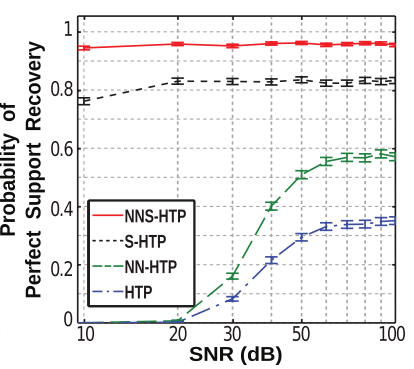

(d) HTP Algorithms

Figure 2: The empirical probability of perfect support recovery as a function of SNR $(d B)$ for the second set of simulations with $K=8$. The error bars in each plot represent $95 \%$ confidence intervals. 


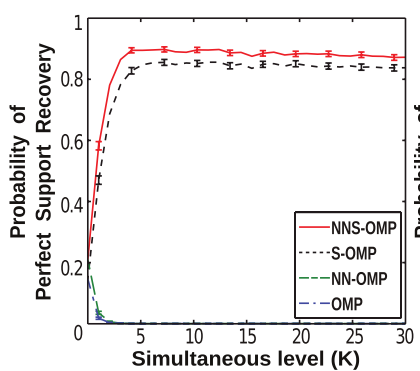

(a) OMP Algorithms

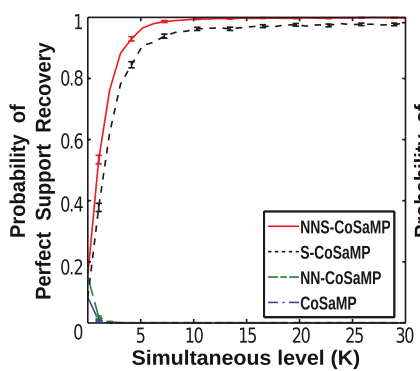

(c) CoSaMP Algorithms

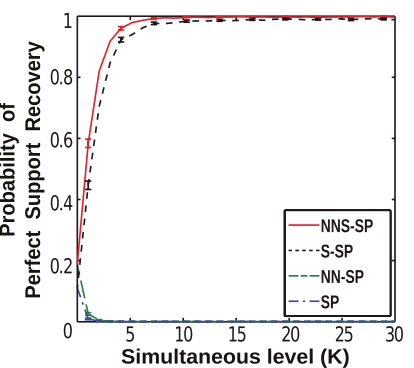

(b) SP Algorithms

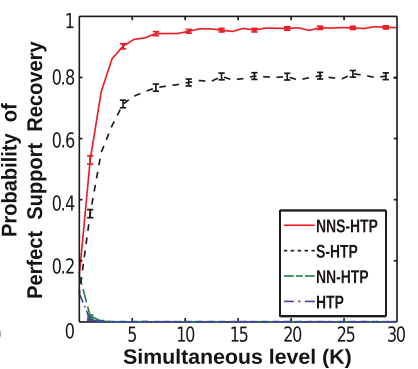

(d) HTP Algorithms

Figure 3: The empirical probability of perfect support recovery as a function of the simultaneous level $K$ for the third set of simulations with SNR $=10 d B$. The error bars in each plot represent $95 \%$ confidence intervals. 


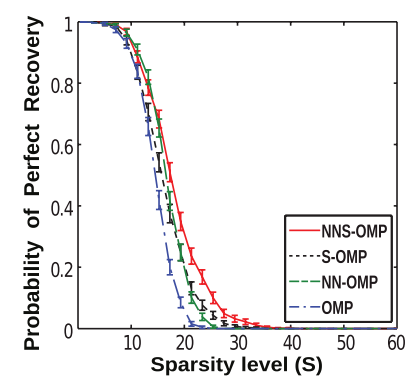

(a) OMP Algorithms

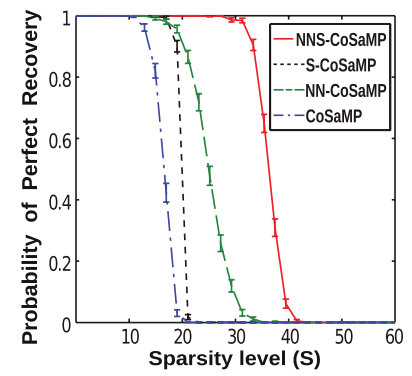

(c) CoSaMP Algorithms

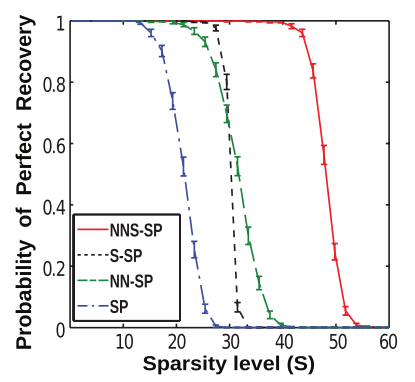

(b) SP Algorithms



(d) HTP Algorithms

Figure 4: The empirical probability of perfect recovery as a function of the sparsity level $S$ for the noiseless fourth set of simulations. The error bars in each plot represent $95 \%$ confidence intervals. 


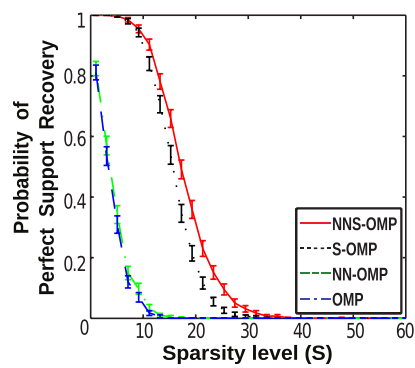

(a) OMP, SNR $=30 \mathrm{~dB}$

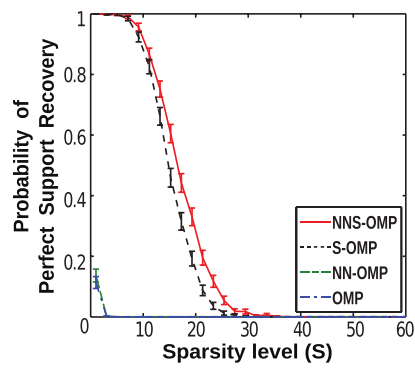

(e) OMP, SNR $=10 \mathrm{~dB}$

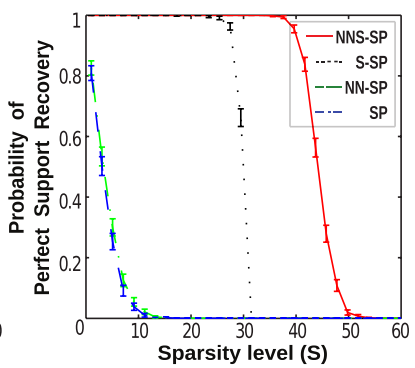

(b) $\mathrm{SP}, \mathrm{SNR}=30 \mathrm{~dB}$

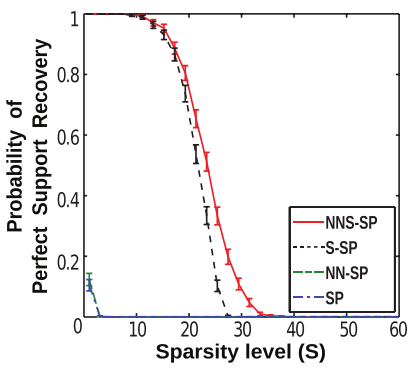

(f) $\mathrm{SP}, \mathrm{SNR}=10 \mathrm{~dB}$

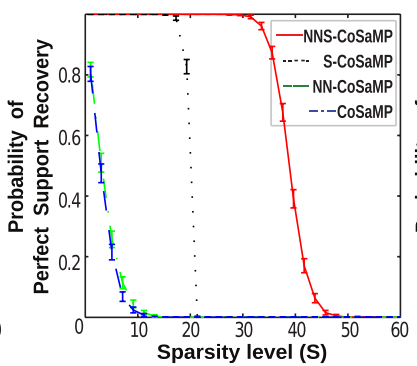

(c) CoSaMP, SNR $=30 \mathrm{~dB}$



(g) CoSaMP, SNR $=10 \mathrm{~dB}$



(d) HTP, SNR $=30 \mathrm{~dB}$

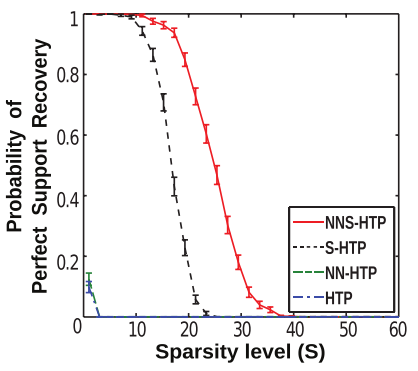

(h) HTP, SNR $=10 \mathrm{~dB}$

Figure 5: The empirical probability of perfect support recovery as a function of the sparsity level $S$ for the fifth set of simulations with noisy signal. (a)-(d) SNR $=30 d B,(e)-(f)$ SNR $=10 d B$. The error bars in each plot represent $95 \%$ confidence intervals. 
Table 1: Mean iterations for different algorithms with $S=4$

\begin{tabular}{lllllll}
\hline & \multicolumn{6}{c}{ Simultaneous level $(K)$} \\
\cline { 2 - 7 } & 5 & 10 & 15 & 20 & 25 & 30 \\
\hline \hline SP & 3.66 & 3.65 & 3.63 & 3.64 & 3.65 & 3.68 \\
NN-SP & 3.47 & 3.50 & 3.45 & 3.49 & 3.47 & 3.51 \\
S-SP & 3.29 & 3.20 & 3.15 & 3.20 & 3.25 & 3.21 \\
NNS-SP & 3.22 & 3.12 & 3.15 & 3.14 & 3.12 & 3.15 \\
\hline \hline CoSaMP & 4.52 & 4.60 & 4.55 & 4.56 & 4.57 & 4.57 \\
NN-CoSaMP & 3.43 & 3.43 & 3.40 & 3.41 & 3.40 & 3.44 \\
S-CoSaMP & 4.27 & 4.21 & 4.17 & 4.12 & 4.14 & 4.30 \\
NNS-CoSaMP & 3.17 & 3.14 & 3.19 & 3.20 & 3.16 & 3.21 \\
\hline \hline HTP & 4.78 & 4.73 & 4.73 & 4.73 & 4.75 & 4.73 \\
NN-HTP & 4.65 & 4.66 & 4.60 & 4.64 & 4.63 & 4.66 \\
S-HTP & 4.39 & 4.33 & 4.37 & 4.35 & 4.47 & 4.25 \\
NNS-HTP & 4.31 & 4.15 & 4.19 & 4.21 & 4.27 & 4.26 \\
\hline
\end{tabular}

Note: The number of iterations for all OMP algorithms is $S=4$. 


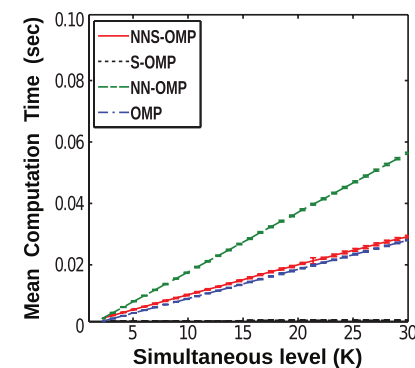

(a) OMP Algorithms

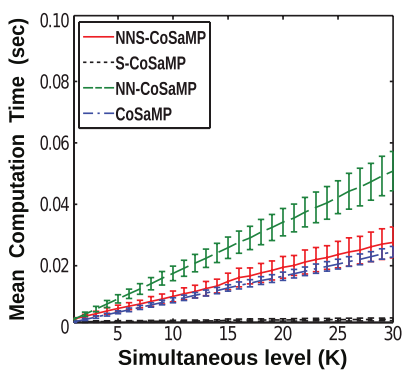

(c) CoSaMP Algorithms

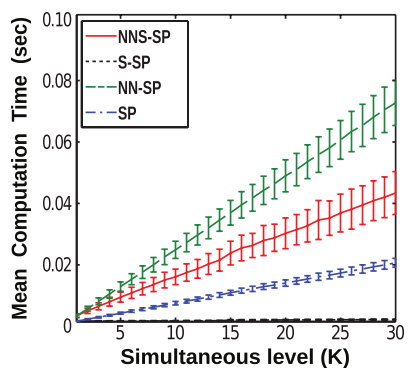

(b) SP Algorithms

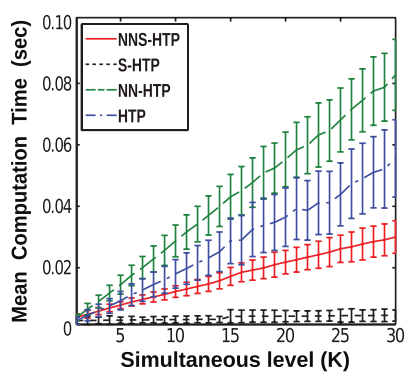

(d) HTP Algorithms

Figure 6: Mean computation time as a function of the simultaneous level $K$. The error bars in each plot represent standard deviations of the computation time. 


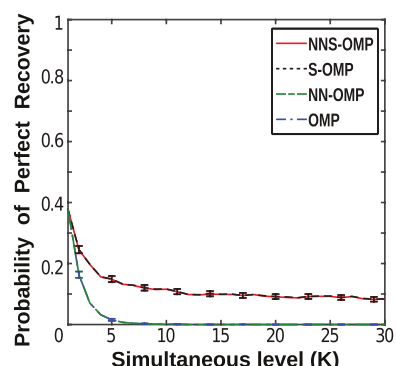

(a) OMP, Y $=\Phi \mathrm{X}$

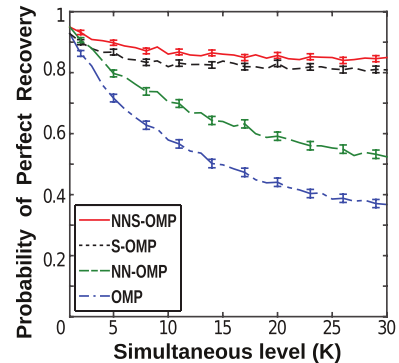

(e) $\mathrm{OMP}, \mathrm{CY}=\mathrm{C} \Phi \mathrm{X}$

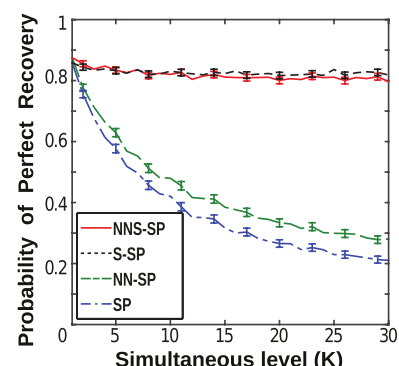

(b) SP, Y $=\Phi X$

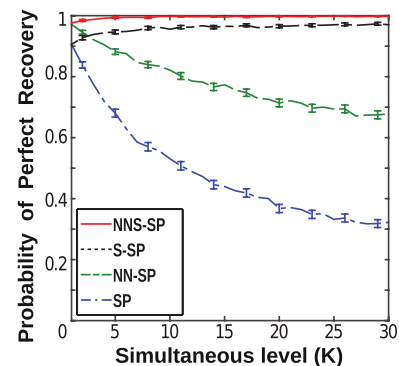

(f) $\mathrm{SP}, \mathrm{CY}=\mathrm{C} \Phi \mathrm{X}$

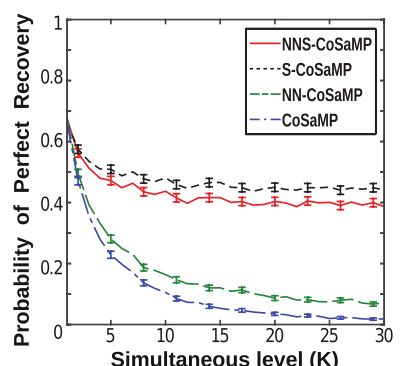

(c) CoSaMP, $\mathrm{Y}=\Phi \mathrm{X}$

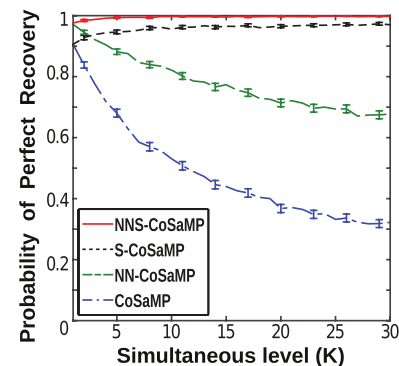

(g) CoSaMP, CY = CФX

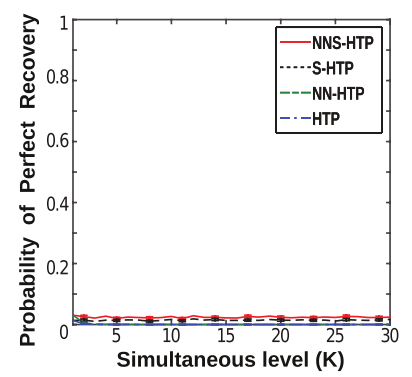

(d) HTP, Y = ФX

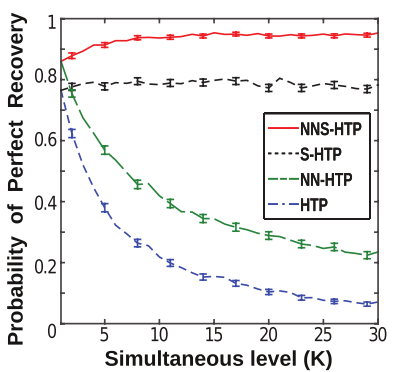

(h) HTP, CY = CФX

Figure 7: The empirical probability of perfect recovery as a function of $K$ for the simulations using Rayleigh matrices $\boldsymbol{\Phi}$. Results are plotted for SS $\mathbf{X}$ matrices estimated from (a)-(d) the original linear model $\mathbf{Y}=\mathbf{\Phi} \mathbf{X}$ and (e)-(h) the conditioned linear model $\mathbf{C Y}=\mathbf{C} \boldsymbol{\Phi} \mathbf{X}$. The entries of $\mathbf{X}$ were drawn iid from a Rayleigh distribution. Results are shown for (a,e) OMP-based algorithms, (b,f) SP-based algorithms, (c,g) CoSaMP-based algorithms, and (d,h) HTP-based algorithms. The error bars in each plot represent $95 \%$ confidence intervals. 

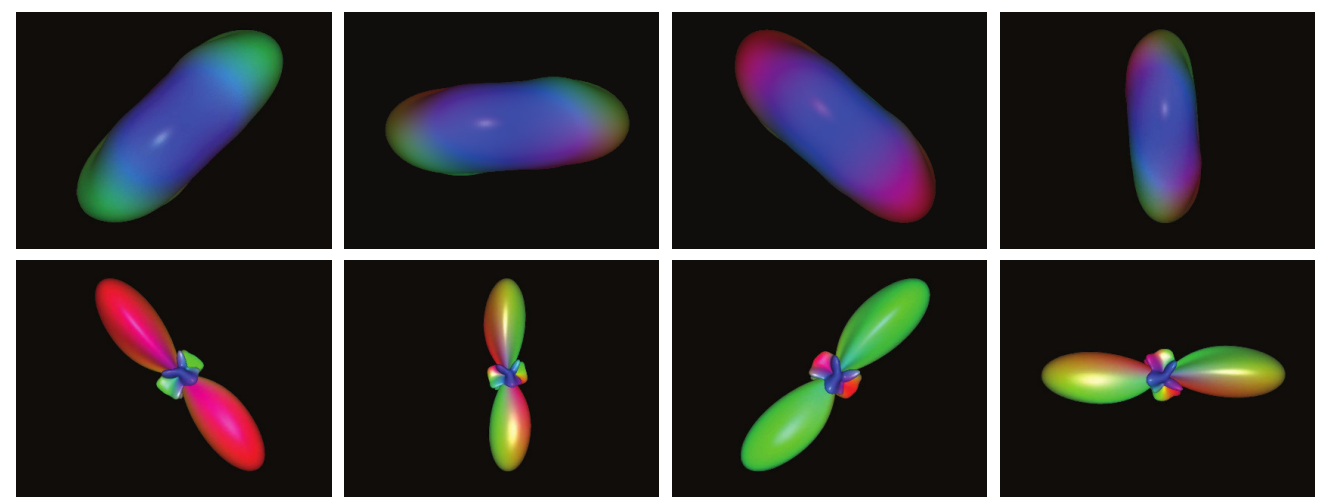

Figure 8: (Top Row) Example diffusion basis functions, which are all resampled rotations of the same basic prototype function. (Bottom Row) ODFs, computed using the Funk-Radon and Cosine Transform [51, corresponding to the diffusion basis functions from the top row. The locations of the largest maximum of each ODF indicate the primary orientations of the corresponding fiber bundle. The diffusion signals and the ODFs have been color-coded by orientation. 




(a) $\mathrm{SP}$

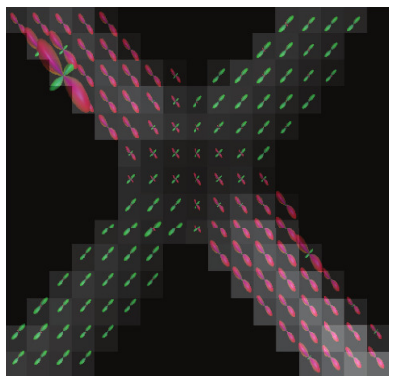

(c) S-SP

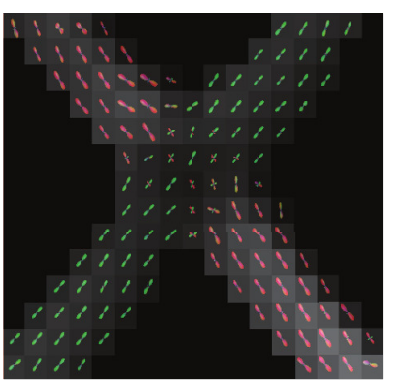

(b) NN-SP

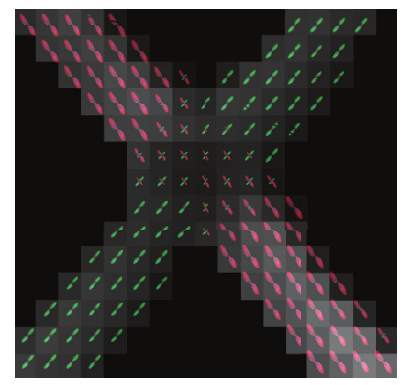

(d) NNS-SP

Figure 9: ODFs estimated using (a) standard SP, (b) NN-SP, (c) S-SP and (d) NNS-SP. 